\title{
Phase 1 Parkinson's Disease Studies Show the Dopamine D1/D5 Agonist PF-06649751 is Safe and Well Tolerated
}

\author{
U. Shivraj Sohur · David L. Gray · Sridhar Duvvuri · Yao Zhang • \\ Kathleen Thayer · Gang Feng
}

Received: July 5, 2018 / Published online: October 25, 2018

(C) The Author(s) 2018

\section{ABSTRACT}

Introduction: There is a need for new therapies in Parkinson's disease that may help to address known limitations of current options. PF06649751 is a novel, highly selective dopamine D1/D5 agonist targeted for Parkinson's disease treatment.

Methods: The safety, pharmacokinetics, and pharmacodynamics of PF-06649751 were assessed in single ascending dose and multiple ascending dose clinical trials in patients with Parkinson's disease. The single ascending dose study $(N=18)$ was a double-blind, placebocontrolled study with a three-way crossover design consisting of three treatment periods separated by 7-day study drug washout periods.

Enhanced digital features To view enhanced digital features for this article go to https://doi.org/10.6084/ m9.figshare.7195070.

Electronic supplementary material The online version of this article (https://doi.org/10.1007/s40120018-0114-z) contains supplementary material, which is available to authorized users.

U. S. Sohur ( $₫)$ · D. L. Gray · S. Duvvuri · Y. Zhang . G. Feng

Pfizer Worldwide Research and Development, Cambridge, MA, USA

e-mail: ussohur@gmail.com

\section{K. Thayer}

Pfizer Global Product Development, New York, NY, USA
PF-06649751 doses were $0.75 \mathrm{mg}, 1.5 \mathrm{mg}, 3 \mathrm{mg}$, $6 \mathrm{mg}$, and $9 \mathrm{mg}$. In the open-label multiple ascending dose study, eligible subjects received once-daily doses of PF-06649751 $(N=45)$ over 21 days, with up-titration to $5 \mathrm{mg}, 15 \mathrm{mg}$, and $25 \mathrm{mg}$ once daily. Pharmacodynamics were assessed by measuring change from baseline in the Movement Disorder Society-sponsored revision of the Unified Parkinson's Disease Rating Scale (MDS-UPDRS) Part III at different time points post dose.

Results: PF-06649751 was safe and well tolerated across studies and in all cohorts. Peak plasma concentrations were attained $1-4 \mathrm{~h}$ post dose across both studies, and exposure increased with increasing dose. PF-06649751 demonstrated sustained pharmacodynamic effects compared with placebo, with mean reductions from baseline in the MDS-UPDRS Part III up to $12 \mathrm{~h}$ post dose at $9 \mathrm{mg}$ single dose. MDS-UPDRS Part III changes in the open-label multiple dose study on day 22 also demonstrated sustained pharmacodynamic activity.

Conclusions: PF-06649751 represents a novel therapeutic candidate for Parkinson's disease with an initial safety, tolerability, and pharmacokinetic profile and potential for efficacy that merits further study in larger clinical trials.

Trial registration: These studies are registered at www.clinicaltrials.gov as NCT02373072, NCT02224664.

Funding: Pfizer. 
Keywords: Dopamine D1 receptor; Dopamine D5 receptor; Dopamine receptor agonists; Parkinson's disease

\section{INTRODUCTION}

The cardinal motor symptoms of Parkinson's disease, which affects over 1 million people in the USA alone, arise as a result of deficiency of the dopamine neurotransmitter in the central nervous system [1]. While levodopa (L-dopa), the synthetic version of dopamine, is the mainstay of Parkinson's disease treatment, more than $40 \%$ of subjects receiving L-dopa experience motor fluctuations typically after more than $3-5$ years of therapy $[2,3]$. These motor fluctuations represent a significant source of disability for some individuals and their families $[4,5]$. In addition, other approved drugs, such as the D2/D3 agonists (e.g., pramipexole and ropinirole), are associated with specific adverse effects, such as daytime somnolence and impulse control disorders [6, 7], that can affect the quality of life of some patients using these medications. Thus, there is an unmet medical need for effective therapies that may be used either as alternatives to, or in combination with, existing Parkinson's disease drugs.

There is a strong rationale, from both preclinical and clinical studies, that targeted activation of dopamine D1 receptor subtype signaling may be an important orthogonal strategy to treat Parkinson's disease while mitigating some of the problems associated with existing therapeutics $[8,9]$. Early advancement of this concept focused on the effects of full D1 agonists, and provided an initial proof of principle via demonstration of a strong antiparkinsonian effect on motor symptoms in several single-dose clinical studies $[10,11]$. One study was conducted in individuals with significant existing dyskinesias, and demonstrated both efficacy and also a level of dyskinesias similar to L-dopa [12]. Dose-limiting effects were also noted in these studies [10-12]. The poor tolerability, low oral bioavailability, and short pharmacokinetic half-life of selective D1 agonists which have reached clinical study to date have limited their further development, and no new investigation of selective D1 agonists in PD has been reported for over 20 years $[13,14]$.

PF-06649751 is a highly selective dopamine D1/D5 receptor partial agonist that has a novel non-catechol-based structure. The discovery of this partial D1 activator with good oral pharmacokinetics and brain penetration prompted us to re-examine this potent pharmacology for the symptomatic treatment of Parkinson's disease. Although we were aware of clinical and preclinical studies which reported that partial D1 agonists were not effective in preclinical models of PD or in clinical study [15-17], other literature [18] raised potential caveats with some of the conclusions from early D1 agonist work, and our own studies also showed suboptimal pharmacokinetics of key tools like SKF38393 [19], which we reasoned could have influenced results.

On the basis of our unpublished studies which showed that non-catechol partial D1 agonists were efficacious in a preclinical model of $\mathrm{PD}$, we advanced the selective $\mathrm{D} 1 / 5$ partial activator PF-06649751 to re-investigate the hypothesis that this pharmacology may provide an important new therapeutic option in Parkinson's with a good balance of safety and motor efficacy. Prior to initiating larger clinical studies for more thorough evaluation of this hypothesis, and in light of prior clinical experience with short-acting selective D1 agonists in clinical studies, we first sought to understand the safety, tolerability, and pharmacokinetics of PF-06649751. Healthy subject studies (single and multiple dose studies) with PF-06649751 were associated with nausea and vomiting, even at low doses, precluding evaluation of safety at projected clinically relevant doses (data on file). Many dopaminergic drugs have divergent tolerability profiles in untreated healthy volunteer vs. treated patients, and we proceeded to complete initial single and multiple dose studies of safety and pharmacokinetics in Parkinson's disease patients who were already receiving levodopa therapy. Preliminary assessment of pharmacodynamics of PF-06649751 was also implemented in these studies to build confidence in the hypothesis and also guide dose selection in phase 2 efficacy studies. 
Here we describe the results of two phase I studies (NCT02373072 and NCT02224664) evaluating the safety, tolerability, pharmacokinetics, and pharmacodynamics of single ascending doses or repeated daily doses of PF06649751 in subjects with idiopathic Parkinson's disease.

\section{METHODS}

\section{Study Overview}

A single ascending dose (SAD) study (NCT02373072) was conducted at five sites in the USA and one site in Belgium, and a multiple ascending dose (MAD) study (NCT02224664) was conducted at 10 centers in the USA. Both studies assessed the safety, tolerability, pharmacokinetics, and pharmacodynamics of PF06649751, a novel dopamine D1/D5 partial agonist in development for the treatment of Parkinson's disease. Participants provided written informed consent prior to screening, and institutional review boards/independent ethics committees at each of the investigational sites reviewed and approved the study protocols (including amendments) and informed consent documentation. Both studies were conducted in accordance with the Declaration of Helsinki and the International Conference on Harmonisation Good Clinical Practice Guideline.

\section{Study Design}

The SAD study was a randomized, double-blind, sponsor-open, placebo-controlled, placebo-substitution study with three-way crossover design in subjects with idiopathic Parkinson's disease (Table 1). After overnight washout of L-dopa (no L-dopa administration after 8 PM), study drug or placebo was administered once during each of three treatment periods, with an at least 7-day study drug washout phase between treatment periods. For cohort 1, each subject participated in the study for up to 61 days, including a 28-day screening period, three study periods with at least 7 days' washout, and up to 10 days' follow-up. For cohort 2, the 61 days could be extended to account for the mandatory safety reviews before each study period. All subjects in cohort 1 period 1 received either PF-06649751 $(0.75 \mathrm{mg})$ or placebo, and dosing proceeded through two additional periods as outlined in Table 1. The start of cohort 2 was held until safety, tolerability, and pharmacokinetics were evaluated for at least six completed subjects in cohort 1 period 2 (PF-06649751 $1.5 \mathrm{mg}$ or placebo). All subjects in cohort 2 received either a higher starting dose of PF-06649751 (3 mg) or placebo, and dosing for cohort 2 proceeded as outlined in Table 1.

In the open-label MAD study, PF-06649751 was administered once daily (QD) over 21 days (days 3-23) to sequential cohorts of subjects with Parkinson's disease (Table 2). PF-06649751 was up-titrated to achieve the target dose by day 14 (11 days of dosing). The screening period for eligibility was up to 24 days (day -28 to day -4 ) prior to day 0. Each cohort had two study periods. For each cohort, subjects entered in period 1 (days $0-2$ ) and, if they met the criteria, were subsequently enrolled into period 2 (days 3-23) and dosed with PF-06649751. On the basis of results observed in a previous MAD study in healthy subjects, PF-06649751 $5 \mathrm{mg}$ QD was selected as the first target dose, which corresponded to cohort 3 from the original protocol. Therefore, cohorts 1 and 2, which had been planned for lower target doses, were not initiated. Cohorts 3, 4, and 6 targeted doses of $5 \mathrm{mg}, 15 \mathrm{mg}$, and $25 \mathrm{mg}$ QD, respectively, and in each case a separate up-titration scheme was employed to allow for 10 days of dosing at the target dose (days 14-23). Doses could be modified or down-titrated according to emerging safety, tolerability, and pharmacokinetic data. Safety, tolerability, and pharmacokinetic data of cohort 3 were reviewed prior to initiating dosing in cohorts 4 and 5 . Cohort 5 was designed to test doses up to $15 \mathrm{mg}$ QD, specifically in Parkinson's disease patients with L-dopa-induced dyskinesia (LID). In cohort 5, subjects were up-titrated over approximately 8 days (days 3-10) to PF-06649751 $8 \mathrm{mg}$ QD; for the remainder of the treatment period, the dose of PF-06649751 could be further increased on the basis of clinical impression to a maximum of $15 \mathrm{mg}$ QD. Available safety, tolerability, and 
Table 1 SAD study: three-way crossover design

\begin{tabular}{|c|c|c|c|c|c|c|}
\hline$N=9$ & Period 1 & & Period 2 & & Period 3 & \\
\hline \multicolumn{7}{|c|}{ Cohort 1} \\
\hline$n=3$ & $\mathrm{PBO}$ & & $1.5 \mathrm{mg}$ & & $3 \mathrm{mg}$ & \\
\hline$n=3$ & $0.75 \mathrm{mg}$ & & $\mathrm{PBO}$ & & $3 \mathrm{mg}$ & \\
\hline$n=3$ & $0.75 \mathrm{mg}$ & & $1.5 \mathrm{mg}$ & & $\mathrm{PBO}$ & \\
\hline \multicolumn{7}{|c|}{ Cohort 2} \\
\hline$n=3$ & Safety review A & $\mathrm{PBO}$ & Safety Review B & $6 \mathrm{mg}$ & Safety review C & $9 \mathrm{mg}$ \\
\hline$n=3$ & & $3 \mathrm{mg}$ & & $\mathrm{PBO}$ & & $9 \mathrm{mg}$ \\
\hline$n=3$ & & $3 \mathrm{mg}$ & & $6 \mathrm{mg}$ & & $\mathrm{PBO}$ \\
\hline
\end{tabular}

$P B O$ placebo, $S A D$ single ascending dose

pharmacokinetic data up to day 24 for at least five subjects from cohort 4 were reviewed prior to initiating the dosing in cohort 6 . Cohorts 5 and 6 were enrolled simultaneously, and the protocol was designed to allow study closeout once at least six subjects had completed the study in cohort 6 .

During up-titration of PF-06649751 in period 2 in the MAD study, there was concomitant gradual down-titration of L-dopa, with the option of L-dopa rescue therapy as determined by the study investigators (Table 2). Subjects who received rescue therapy with L-dopa continued in the study. At the end of period 2, subjects could return to their pre-study doses of anti-Parkinson's disease medications.

\section{Inclusion/Exclusion Criteria}

Both the SAD and MAD studies admitted male or female subjects of non-childbearing potential between the ages of 30 and 85 years (SAD study) or 30 and 80 years (MAD study), inclusive, with a body mass index of $17.5-38.0 \mathrm{~kg} / \mathrm{m}^{2}$ and a total body weight greater than $50 \mathrm{~kg}$, and with a clinical diagnosis of idiopathic Parkinson's disease, including the presence of at least two out of three cardinal characteristics (tremor, rigidity, and/or bradykinesia). For both studies, subjects were excluded if they had a history of or clinical features consistent with an atypical parkinsonian syndrome, or a history of surgical intervention for Parkinson's disease (pallidotomy, thalamotomy, deep brain stimulation, etc.).

In the MAD study, amantadine (except cohort 5), apomorphine, anticholinergics, catechol-O-methyl transferase inhibitors, and dopamine agonists were not permitted at any time (days 0-24). Amantadine (except cohort 5), apomorphine, and anticholinergics were to be discontinued at least 21 days prior to day 0 . Catechol-O-methyl transferase inhibitors and dopamine agonists were to be discontinued on day -3 (or prior). Monoamine oxidase inhibitors were allowed throughout the study, with the exception of intensive pharmacokinetic/pharmacodynamic days. Cohort 5 only: daily doses of amantadine up to $200 \mathrm{mg} /$ day were allowed throughout the study; daily doses of amantadine were to be stable for at least 21 days prior to day 0.

\section{Study Objectives}

The primary objective of these studies was to evaluate the safety and tolerability of PF06649751 in subjects with Parkinson's disease. The pharmacokinetics of PF-06649751 was a secondary objective. Additionally, in both studies, we sought to explore the pharmacodynamic effects of PF-06649751 on motor performance and dyskinesia using the Movement Disorder Society-sponsored revision of the Unified Parkinson's Disease Rating Scale Part III (MDS-UPDRS-III) [20]. 


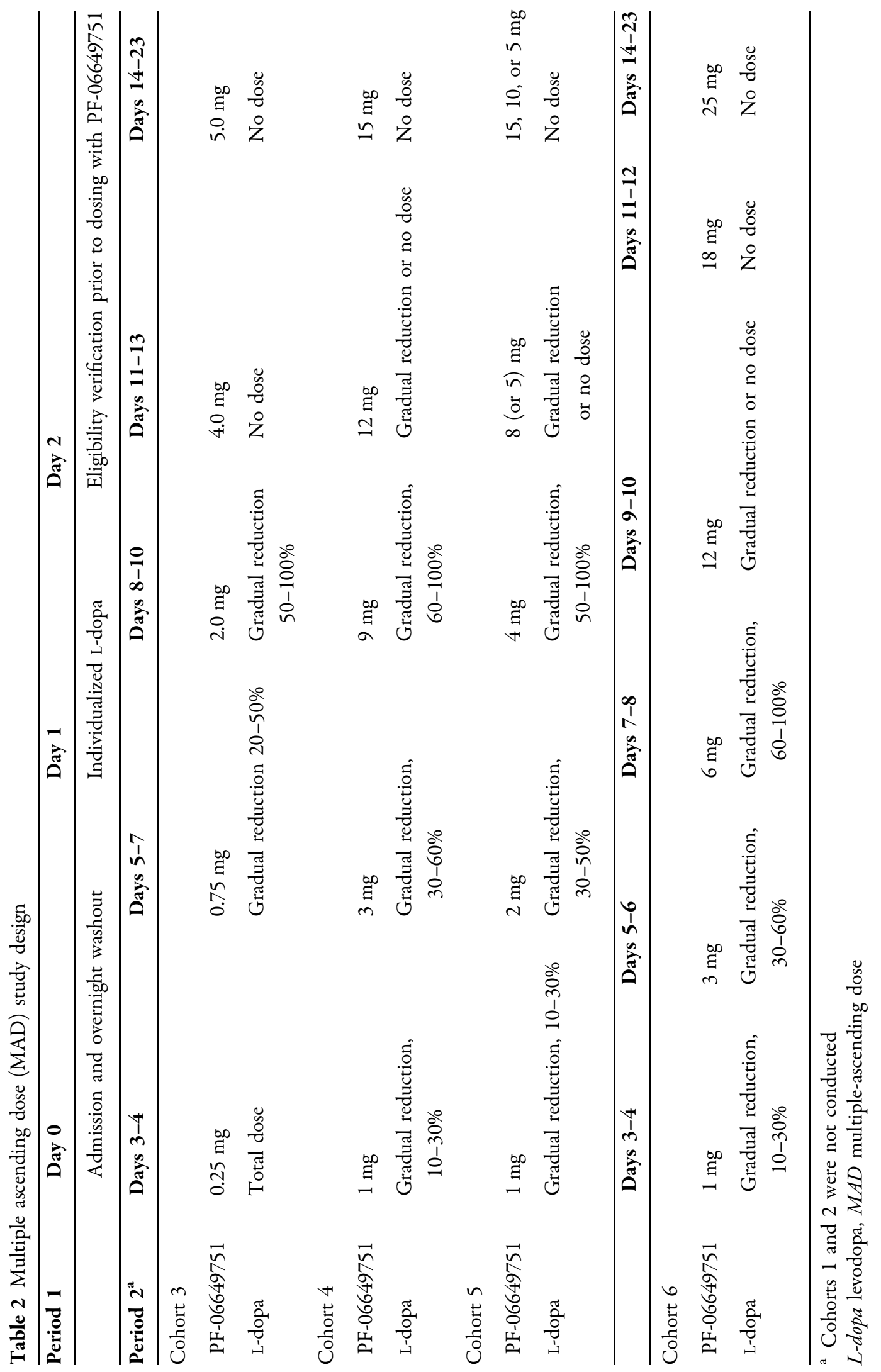




\section{MDS-UPDRS-III Evaluation}

The MDS-UPDRS is a structured clinimetric tool used to evaluate the severity of Parkinson's disease across behaviors, daily activities, motor abilities, and other complications of Parkinson's disease [20]. The MDS-UPDRS primarily measures motor impairments, with subsections organized according to motor and non-motor aspects of Parkinson's disease.

The MDS-UPDRS-III assesses the motor signs of Parkinson's disease and was administered by the study investigators, who maintained current training and certification on the instrument. All Part III assessments were performed by the local rater and, in the SAD study, rating sessions were video-recorded for evaluation by an independent central qualified rater. MDSUPDRS-III comprises 33 subscores based on 18 items, several with right, left, or other body distribution scores. Each question was anchored with five responses that were linked to commonly accepted clinical terms: $0=$ normal, $1=$ slight, $2=$ mild, $3=$ moderate, and $4=$ severe. In the MAD study, MDS-UPDRS-III assessments took place each study day and at intervals of $0.5,1,1.5,2,4,8$, and $12 \mathrm{~h}$ on intensive pharmacokinetic/pharmacodynamic study days (days 7,13 , and 22). L-Dopa was not administered until after pharmacokinetic and pharmacodynamic assessments were completed, except where rescue L-dopa was required, in which case data from these subjects were excluded from the pharmacodynamic analysis.

\section{Safety Evaluations}

Safety evaluations included adverse events (AEs), safety laboratory tests, vital signs, 12-lead electrocardiograms (ECGs), physical and neurological examinations, and suicidal ideation and behavior assessments including ColumbiaSuicide Severity Rating Scale.

\section{Statistical Methods}

A sample size of approximately nine (SAD study) and eight (MAD study) completers per cohort was chosen on the basis of the need to minimize exposure of humans to a new chemical entity and the requirement to provide adequate safety and tolerability information at each dose.

In the SAD study, change from baseline in MDS-UPDRS-III total score was analyzed using a restricted maximum likelihood linear mixedeffect repeated measures model with fixed effects for treatment, time (visit), baseline MDSUPDRS-III total motor score, and a random effect for subject. Using this model, 90\% confidence intervals (CIs) comparing the mean change from baseline in MDS-UPDRS-III total motor score estimates at each time point for PF06649751 versus placebo were computed. Analysis was to be done on the highest achieved PF-06649751 dose along with the matching placebo data for that period. If the highest PF06649751 dose was at least $3 \mathrm{mg}$, then analysis was also to be done for the PF-06649751 $3 \mathrm{mg}$ dose versus placebo, with data pooled across the two cohorts.

In the MAD study, no formal inferential statistics were applied to the pharmacodynamic analysis, and results were descriptive only, with comparisons generally made to baseline, as appropriate. Descriptive statistics over time were used to detect trends via changes within the treatment groups or differences between groups, if applicable.

\section{RESULTS}

In the SAD study, a total of 18 subjects were randomized and assigned to study treatments. All subjects completed the treatment, except for one subject from cohort 2 who discontinued after two periods as they were no longer willing to participate. The subject received treatment with PF-06649751 $3 \mathrm{mg}$ and PF-06649751 $6 \mathrm{mg}$ but did not receive the placebo treatment. All subjects treated with PF-06649751 were included in the pharmacokinetic analysis. One subject (PF-06649751 9 mg) had a pre-dose concentration of $9.5 \%$ of peak concentration $\left(C_{\max }\right)$ and was included in the analysis. All subjects treated with study drugs were analyzed for pharmacodynamics and safety. The majority 
of the subjects were male (15/18) and white (16/ $18)$. The mean age (60.1 years, range $44-$ 76 years $)$ mean weight $(81.0 \mathrm{~kg}$, range $56.1-126.6 \mathrm{~kg})$, and body mass index $(27.8 \mathrm{~kg} /$ $\mathrm{m}^{2}$, range $18.3-37.5 \mathrm{~kg} / \mathrm{m}^{2}$ ) were generally comparable across the two cohorts (Supplementary Table 1). For the MAD study, a total of 50 subjects were assigned to study treatment (Ldopa) in period 1 . Of the 50 subjects, 45 subjects met the criteria for enrollment to period 2, and five subjects were discontinued from the study as they did not meet the period 2 entrance criteria. Forty-five subjects distributed across cohorts 3-6 were treated with PF-06649751. Of the 50 subjects in the study, 31 were male and 19 were female; the majority of the subjects were white (44/50 subjects) and the age range was 44-76 (Supplementary Table 2).

\section{Pharmacokinetics}

\section{SAD Study}

Following administration of single oral doses of PF-06649751 from $0.75 \mathrm{mg}$ to $9 \mathrm{mg}$ under fasted conditions, PF-06649751 $C_{\max }$ occurred at approximately $3.0-4.0 \mathrm{~h}$ post dose (Supplementary Fig. 1 top panel and Supplementary Table 3$)$. The half-life $\left(t_{1 / 2}\right)$ of PF-06649751 for the 3-mg group in cohort 2 was approximately $16 \mathrm{~h}$. The $t_{1 / 2}$ was not reportable for most treatments because the data were not sufficient for adequate characterization of the elimination phase.

Both peak exposure $\left(C_{\max }\right)$ and total exposure $\left(A U C_{\text {last }}\right)$ increased with increasing dose from $0.75 \mathrm{mg}$ to $9 \mathrm{mg}$, and the increases appeared to be dose proportional as evidenced by similar dose-normalized $\mathrm{AUC}_{\text {last }}$ and $C_{\max }$ across all doses. Inter-subject variability for PF06649751 exposure, based on geometric percent coefficient of variation (\%CV), ranged from $21 \%$ to $44 \%$ for $\mathrm{AUC}_{\text {last }}$ and $26 \%$ to $42 \%$ for $C_{\text {max }}$ across all treatments.

\section{MAD Study}

Median plasma PF-06649751 concentration-time profiles following multiple-dose administration on day 22 are presented in Supplementary Fig. 1 (bottom panel).
Pharmacokinetic parameters for day 22 are summarized descriptively in Supplementary Table 4. On day 22 (steady-state), following administration of multiple oral doses of PF06649751 from $5 \mathrm{mg}$ to $25 \mathrm{mg}$, mean $C_{\max }$ for PF-06649751 occurred at approximately 2.0-4.0 $\mathrm{h}$ post dose. The PF-06649751 exposures (geometric means of $C_{\max }$ and $\mathrm{AUC}_{\mathrm{tau}}$ ) increased with an increase of dose. On the basis of average values in Parkinson's disease subjects with motor fluctuations (cohort 4 vs cohort 6 ), the increase in $\mathrm{AUC}_{\text {tau }}$ was less than dose proportional between $15 \mathrm{mg}$ and $25 \mathrm{mg}$. The mean apparent clearance values ranged from $2.4 \mathrm{~L} / \mathrm{h}$ to $3.5 \mathrm{~L} / \mathrm{h}$. On day 22 , inter-subject variability for PF-06649751 exposure, based on geometric $\% \mathrm{CV}$, ranged from $35 \%$ to $61 \%$ for $\mathrm{AUC}_{\text {tau }}$ and $26 \%$ to $46 \%$ for $C_{\max }$ across all cohorts.

\section{Pharmacodynamics}

In the SAD study, there was a statistically significant $(p<0.05)$ decrease in least squares (LS) mean change from baseline in MDS-UPDRS-III total motor score on day 1 at $1,2,4,8$, and $12 \mathrm{~h}$ in subjects in the PF-06649751 9-mg treatment group compared with placebo (Fig. 1). At $12 \mathrm{~h}$ post dose the LS mean change of MDS-UPDRSIII scores from baseline was $-0.82 \pm 3.26(90 \%$ $\mathrm{CI},-6.35,4.71)$ and $-11.96 \pm 4.16(90 \% \mathrm{CI}$, $-18.88,-5.03)$ for placebo and PF-06649751 $9 \mathrm{mg}$, respectively. The MDS-UPDRS-III demonstrated a statistically significant difference of $-11.13 \pm 3.68$ (90\% CI, $-17.21,-5.06$; $p=0.0028$ ) in the PF-06649751 9-mg treatment group versus placebo. Similarly, there were greater decreases from baseline in LS mean total motor score on day 1 at $1,2,4,8$, and $12 \mathrm{~h}$ in subjects in the PF-06649751 3-mg treatment group versus placebo; however, the differences were not statistically significant. Arithmetic mean changes from baseline in MDS-UPDRS-III subscale scores are also shown in Fig. 1.

In the MAD study, a sustained reduction of MDS-UPDRS-III scores was observed on day 22 of cohorts 4 and 6 (PF-06649751 $15 \mathrm{mg}$ and $25 \mathrm{mg}$ ) (Fig. 2, top panel, and Table 3). On day 22 , at $12 \mathrm{~h}$ post dose the mean change of MDSUPDRS-III scores from baseline was $2.25 \pm 6.50$ 

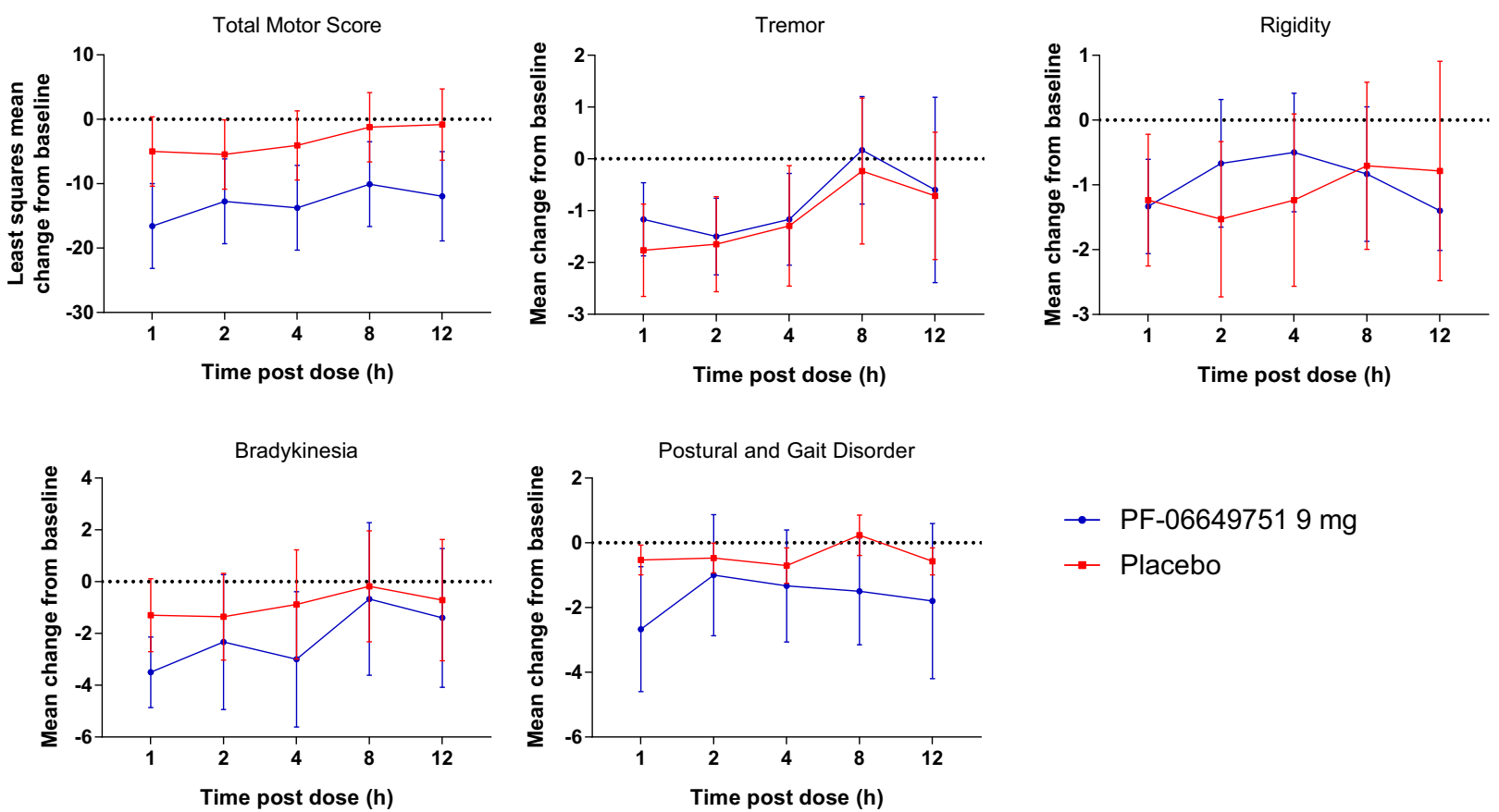

$\rightarrow$ PF-066497519 mg
$\rightarrow-$ Placebo

Fig. 1 Mean change from baseline in MDS-UPDRS-III total score [least-squares mean $(90 \% \mathrm{CI})$ ] and subscale scores [arithmetic mean $(80 \% \mathrm{CI})$ ] at day 1 in the SAD study $9 \mathrm{mg}$ cohort. CI confidence interval, SAD single

(80\% CI, $-1.0,5.50),-20.0 \pm 12.90(80 \% \mathrm{CI}$, $-27.02,-12.98)$, and $-9.33 \pm 14.60(80 \% \mathrm{CI}$, $-18.13,-0.54$ ) for cohorts 3,4 , and 6 (PF$066497515 \mathrm{mg}, 15 \mathrm{mg}$, and $25 \mathrm{mg}$ ), respectively. In addition, 44\% (4/9), 78\% (7/9), and $50 \%(4 / 8)$ of the completed subjects in cohorts 3 (PF-06649751 $5 \mathrm{mg}$ ), 4 (PF-06649751 $15 \mathrm{mg}$ ), and 6 (PF-06649751 $25 \mathrm{mg}$ ), respectively, experienced more than 10 L-dopa-free days (Fig. 2, bottom panel). There was a lack of an observed pharmacodynamic effect in cohort 5 (PF06649751 LID), although numbers in this cohort were small $(n=3)$. It is important to note that the MAD study was conducted in an open-label fashion; therefore data should be interpreted accordingly.

\section{Safety}

In the SAD study, single doses of PF-06649751 up to $9 \mathrm{mg}$ were safe and well tolerated by subjects with Parkinson's disease. There were no deaths, serious AEs (SAE), severe AEs, discontinuations due to AEs, dose reductions, or ascending dose, MDS-UPDRS-III Movement Disorder Society-sponsored revision of the Unified Parkinson's Disease Rating Scale Part III

temporary discontinuations due to AEs. A total of 46 all-causality treatment-emergent AEs (TEAEs) were reported, of which 35 were considered treatment related. All TEAEs were mild to moderate in severity (Supplementary Table 5). The most common TEAEs across all groups were headache, nausea, and vomiting (Supplementary Table 6). There appeared to be a small dose-related increase in QT interval corrected for heart rate using Fridericia's formula (QTcF) at the 2, 4, and $8 \mathrm{~h}$ post dose time points. The greatest increase from baseline in mean QTcF at any time point for any dose group was $11.7 \mathrm{~ms}$. Despite these observations in the SAD study, no QTcF prolongation was observed in the MAD study, where higher PF06649751 exposures were achieved, and no subject had a QTcF $\geq 500 \mathrm{~ms}$ or an increase of $\mathrm{QTcF} \geq 60 \mathrm{~ms}$.

In the MAD study, a total of 172 all-causality AEs were reported. Cohort 3 had the highest proportion of subjects experiencing AEs, with all nine subjects in the cohort experiencing a total of 43 AEs, followed by cohort 5 , in which 

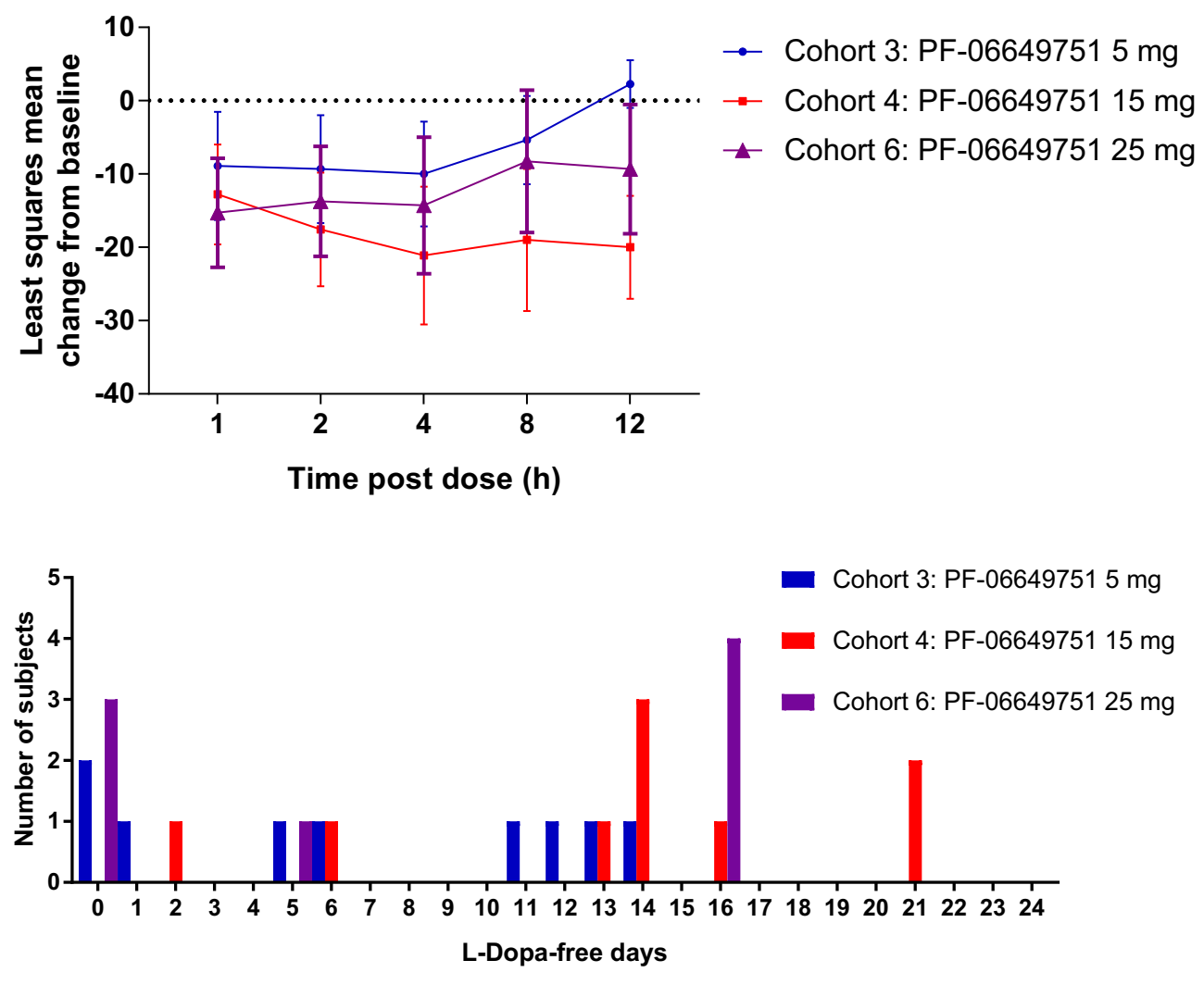

Fig. 2 Top panel: least squares mean (80\% CI) change from baseline in MDS-UPDRS-III total score at day 22 in the MAD study. Bottom panel: number of L-dopa-free days in the MAD study. CI confidence interval, SAD

five of six subjects experienced 27 AEs (Supplementary Table 5). Eleven subjects permanently discontinued the study owing to AEs: two in cohort 4 (PF-06649751 $15 \mathrm{mg}$ ), two in cohort 5 (PF-06649751 LID), and seven in cohort 6 (PF$0664975125 \mathrm{mg}$ ). The most common AEs reported in all PF-06649751 treatment groups were headache, nausea, abnormal dreams, dizziness, and vomiting (Supplementary Table 6). The majority of the AEs were mild to moderate in severity, except for six AEs that were severe in nature. AEs in all treatment groups occurred in the up-titration phase (days 3-24 and follow-up) and appeared related to pace and increment of up-titration rather than maximum exposure, and they were generally self-limited. There was only one SAE (palpitations), which occurred in cohort 6 on study day 3 when the PF-06649751 dose was $1 \mathrm{mg}$. Study single ascending dose, MDS-UPDRS-III Movement Disorder Society-sponsored revision of the Unified Parkinson's Disease Rating Scale Part III

drug was permanently discontinued and the SAE resolved 3 days later. This SAE was not considered by the investigator to be treatment related. No deaths occurred during the study. For both studies, there were no notable findings in clinical laboratory, physical, and neurological examinations, vital signs, or in suicidal ideation and behavior assessments.

\section{DISCUSSION}

Activation of the dopamine signaling pathway remains a target for the treatment of Parkinson's disease. In these two studies we assessed the safety, tolerability, and pharmacokinetics of a novel oral dopamine D1/D5 agonist in subjects with idiopathic Parkinson's disease.

Results show that multiple doses of PF06649751 up to $25 \mathrm{mg}$ were generally safe and 


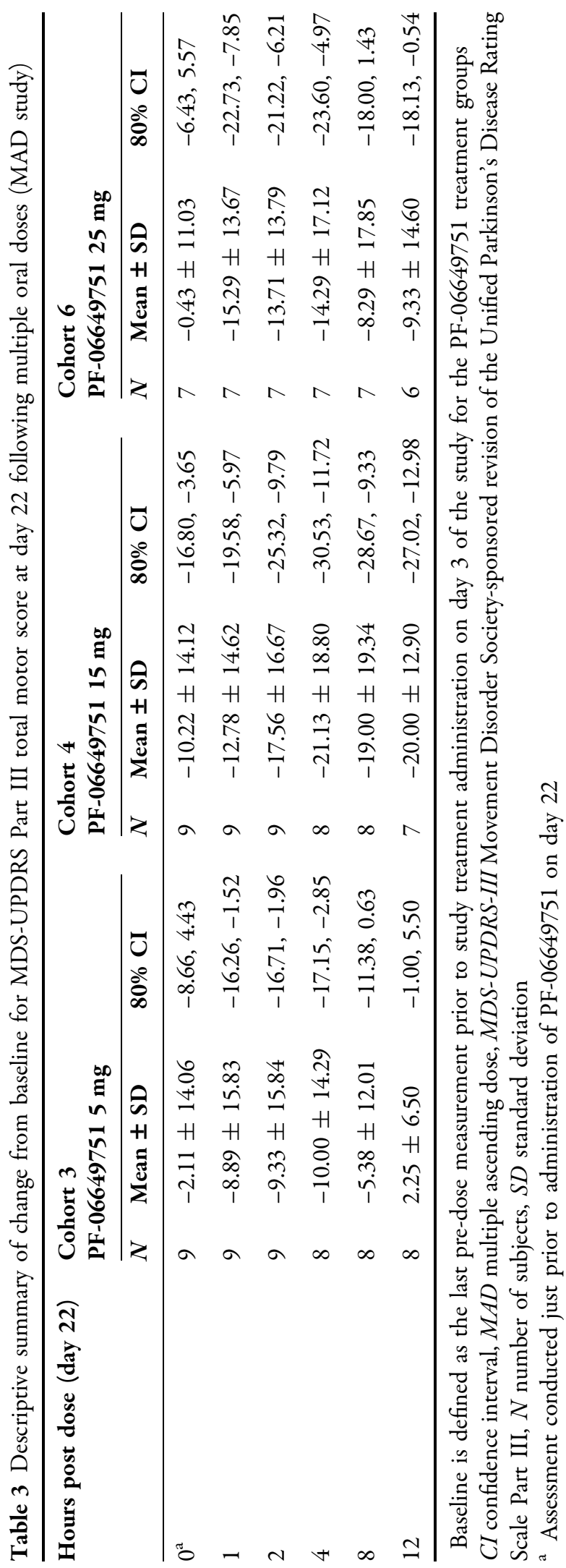

well tolerated in subjects with idiopathic Parkinson's disease, and single doses of PF06649751 up to $9 \mathrm{mg}$ were safe and well tolerated in the SAD study, suggesting that a relatively short titration period may be feasible. Notably, the single PF-06649751 9 mg dose was also associated with statistically significant improvements versus placebo in motor function determined by total motor score on the MDSUPDRS. Across studies and cohorts, there were no apparent trends or clinically significant changes in vital signs, ECG, physical findings, laboratory values, or suicidal ideation or behavioral assessments. In the single-dose study there was an increase of mean QTcF values in the higher-dose groups (PF-06649751 $6 \mathrm{mg}$ and PF-06649751 $9 \mathrm{mg}$ ). The highest mean change in QTcF was $11.7 \mathrm{~ms}$, recorded in the 9-mg dose group. However, in the multiple-dose study, across doses and cohorts, there were no apparent trends or clinically significant changes in ECG, including no changes in QTcF. The reason for observed QTcF changes following single doses requires further investigation as PF-06649751 is expected to interact minimally with hERG (potassium ion) channels at clinically relevant concentrations.

Peak plasma concentrations of PF-06649751 were reached approximately $1-4 \mathrm{~h}$ post dose, and increases in both $C_{\max }$ and $\mathrm{AUC}_{\text {last }}$ appeared to be dose proportional across most of the dose range; however, the increase in mean $\mathrm{AUC}_{\text {tau }}$ was less than dose proportional between the $15 \mathrm{mg}$ (cohort 4) and $25 \mathrm{mg}$ (cohort 6) doses in the MAD study.

In terms of efficacy or pharmacodynamics for the SAD study, there were statistically significant decreases from baseline in LS mean total motor score on day 1 at $1,2,4,8$, and $12 \mathrm{~h}$ in subjects treated with PF-06649751 $9 \mathrm{mg}$ versus placebo. Similarly, decreases were seen in the PF-06649751 3-mg group, but these were not statistically significant compared with placebo. In the MAD study, PF-06649751 demonstrated sustained pharmacodynamic effects, with group mean MDS-UPDRS-III scores around 9-20 points below baseline for assessments conducted at $12 \mathrm{~h}$ post dose on day 22 in cohort 4 (15 mg QD) and cohort 6 (25 mg QD) (Table 3). Pre-dose reductions were evident at time 0 on day 22 in cohort 4 (15 mg QD), 
suggesting a sustained effect of previous dosing (Table 3). There were too few subjects in cohort 5 (LID) to evaluate the effects of PF-06649751 dosing on LID or overall reductions in L-dopa dosing during the cross-titration phase; however, data from other cohorts showed that $44 \%$, $78 \%$, and $50 \%$ of the completed subjects in cohorts 3 (5 mg QD), 4 (15 mg QD), and 6 (25 mg QD), respectively, experienced more than 10 L-dopa-free days.

The multi-dose study was conducted in an open-label fashion, which does not facilitate direct and quantitative comparison of efficacy results with established agents. While the data from these studies are encouraging, it should be noted that larger blinded studies or studies of longer duration will be required to adequately investigate potential efficacy and safety. The relatively short duration of the studies described herein can only provide limited data on potential efficacy and address acute rather than long-term safety. On the basis of these results, a range of 1-15 $\mathrm{mg}$ QD represents the possible therapeutic range for PF-06649751 for further evaluation in phase $2 \mathrm{~b}$ dose ranging studies to facilitate final dose selection.

\section{CONCLUSION}

In summary, these studies suggest that PF06649751 is worthy of further clinical investigation as a treatment for Parkinson's disease. Larger-scale phase II clinical trials (NCT03185481, NCT02687542, NCT02847650) have been initiated to assess the potential safety and efficacy of this novel oral dopamine D1/D5 agonist for the treatment of Parkinson's disease, and it is hoped that these will help determine a dose of PF06649751 that can lead to sustained reductions in symptoms while reducing dependence on Ldopa, thereby avoiding the problems associated with long-term L-dopa administration.

\section{ACKNOWLEDGEMENTS}

We thank the investigators and all of their staff members who worked on this study, and are particularly grateful to all of the participants with Parkinson's who were screened and or participated in support of this research.

Funding. This study was sponsored by Pfizer. Article processing and open access fees were funded by Pfizer. All authors had full access to all of the data in this study and take complete responsibility for the integrity of the data and accuracy of the data analysis.

Medical Writing and/or Editorial Assistance. Medical writing and editorial assistance was provided by Paul Hassan, PhD, of Engage Scientific Solutions (Horsham, West Sussex, UK) and was funded by Pfizer. We also wish to thank Joel Posener (Pfizer, Cambridge, MA, USA) and Frederick Carter (inVentiv Health, Atlanta, GA, USA) for critical review of the manuscript.

Authorship. All named authors meet the International Committee of Medical Journal Editors (ICMJE) criteria for authorship for this article, take responsibility for the integrity of the work as a whole, and have given their approval for this version to be published.

Author Contributions. All authors contributed to the design and implementation of these studies, the acquisition, analysis, and interpretation of the data, and the development of the manuscript. The decision to submit the manuscript was a collective decision and all authors approved the final version for submission.

Disclosures. David L. Gray is an employee of the study sponsor, Pfizer. Sridhar Duvvuri is an employee of the study sponsor, Pfizer. Yao Zhang is an employee of the study sponsor, Pfizer. Kathleen Thayer is an employee of the study sponsor, Pfizer. Gang Feng is an employee of the study sponsor, Pfizer. U. Shivraj Sohur was an employee of Pfizer during the study and development of this manuscript. U. Shivraj Sohur is now affiliated with Alkermes Pharmaceuticals Research \& Development.

Compliance with Ethics Guidelines. Institutional review boards/independent ethics committees at each of the investigational sites 
reviewed and approved the study protocols (including amendments) and informed consent documentation. Both studies were conducted in accordance with the Declaration of Helsinki and the International Conference on Harmonisation Good Clinical Practice Guideline.

Data Availability. Upon request, and subject to certain criteria, conditions and exceptions (see https://www.pfizer.com/science/ clinical-trials/trial-data-and-results for more information), Pfizer will provide access to individual de-identified participant data from Pfizer-sponsored global interventional clinical studies conducted for medicines, vaccines and medical devices (1) for indications that have been approved in the USA and/or EU or (2) in programs that have been terminated (i.e., development for all indications has been discontinued). Pfizer will also consider requests for the protocol, data dictionary, and statistical analysis plan. Data may be requested from Pfizer trials 24 months after study completion. The deidentified participant data will be made available to researchers whose proposals meet the research criteria and other conditions, and for which an exception does not apply, via a secure portal. To gain access, data requestors must enter into a data access agreement with Pfizer.

Open Access. This article is distributed under the terms of the Creative Commons Attribution-NonCommercial 4.0 International License (http://creativecommons.org/licenses/ by-nc/4.0/), which permits any noncommercial use, distribution, and reproduction in any medium, provided you give appropriate credit to the original author(s) and the source, provide a link to the Creative Commons license, and indicate if changes were made.

\section{REFERENCES}

1. Nussbaum RL, Ellis CE. Alzheimer's disease and Parkinson's disease. N Engl J Med. 2003;348:1356-64.

2. Schrag A, Quinn N. Dyskinesias and motor fluctuations in Parkinson's disease. A community-based study. Brain. 2000;123(Pt 11):2297-305.
3. Bastide MF, Meissner WG, Picconi B, et al. Pathophysiology of L-dopa-induced motor and non-motor complications in Parkinson's disease. Prog Neurobiol. 2015;132:96-168.

4. Olanow CW, Obeso JA, Stocchi F. Continuous dopamine-receptor treatment of Parkinson's disease: scientific rationale and clinical implications. Lancet Neurol. 2006;5:677-87.

5. Olanow CW, Obeso JA, Stocchi F. Drug insight: continuous dopaminergic stimulation in the treatment of Parkinson's disease. Nat Clin Pract Neurol. 2006;2:382-92.

6. Boehringer Ingelheim International $\mathrm{GmbH}$. MIRAPEX prescribing information. March 5, 2018. https://www.accessdata.fda.gov/drugsatfda_docs/ label/2016/020667s034lbl.pdf.

7. GlaxoSmithKline. REQUIP Prescribing Information. March 5, 2018. https://www.accessdata.fda. gov/drugsatfda_docs/label/2017/020658s034lbl. pdf.

8. Waddington JL. Therapeutic potential of selective D-1 dopamine receptor agonists and antagonists in psychiatry and neurology. Gen Pharmacol. 1988;19:55-60.

9. Giardina WJ, Williams M. Adrogolide $\mathrm{HCl}$ (ABT431; DAS-431), a prodrug of the dopamine D1 receptor agonist, A-86929: preclinical pharmacology and clinical data. CNS Drug Rev. 2001;7:305-16.

10. Rascol O, Blin O, Thalamas C, et al. ABT-431, a D1 receptor agonist prodrug, has efficacy in Parkinson's disease. Ann Neurol. 1999;45:736-41.

11. Blanchet PJ, Fang J, Gillespie M, et al. Effects of the full dopamine D1 receptor agonist dihydrexidine in Parkinson's disease. Clin Neuropharmacol. 1998;21:339-43.

12. Rascol O, Nutt JG, Blin $\mathrm{O}$, et al. Induction by dopamine D1 receptor agonist ABT-431 of dyskinesia similar to levodopa in patients with Parkinson disease. Arch Neurol. 2001;58:249-54.

13. Sokoloff P, Schwartz JC. Novel dopamine receptors half a decade later. Trends Pharmacol Sci. $1995 ; 16: 270-5$.

14. Mailman R, Huang X, Nichols DE. Parkinson's disease and D1 dopamine receptors. Curr Opin Investig Drugs. 2001;2:1582-91.

15. Braun A, Fabbrini G, Mouradian MM, Serrati C, Barone P, Chase TN. Selective D-1 dopamine receptor agonist treatment of Parkinson's disease. J Neural Transm. 1987;68:41-50. 
16. Close SP, Marriott AS, Pay S. Failure of SKF 38393-A to relieve parkinsonian symptoms induced by 1-methyl-4-phenyl-1,2,3,6-tetrahydropyridine the marmoset. Br J Pharmacol. 1985;85:320-2.

17. Watts VJ, Lawler CP, Gilmore JH, Southerland SB, Nichols DE, Mailman RB. Dopamine D1 receptors: efficacy of full (dihydrexidine) vs. partial (SKF38393) agonists in primates vs. rodents. Eur J Pharmacol. 1993;242:165-72.

18. Gnanalingham KK, Erol DD, Hunter AJ, Smith LA, Jenner P, Marsden CD. Differential anti-parkinsonian effects of benzazepine D1 dopamine agonists with varying efficacies in the MPTP-treated common marmoset. Psychopharmacology. 1995;117:275-86.

19. Gray DL, Allen JA, Mente S, et al. Impaired betaarrestin recruitment and reduced desensitization by non-catechol agonists of the D1 dopamine receptor. Nat Commun. 2018;9:674.

20. Goetz CG, Fahn S, Martinez-Martin P, et al. Movement disorder society-sponsored revision of the unified Parkinson's Disease rating scale (MDSUPDRS): process, FORMAT, and clinimetric testing plan. Mov Disord. 2007;22:41-7. 